\title{
Bap29varP, a variant of Bap29, influences the cell surface expression of the human P-glycoprotein
}

\author{
PREMA S. RAO, ULRICH BICKEL, KALKUNTE S. SRIVENUGOPAL and U. SUBRAHMANYESWARA RAO \\ The Anticancer Resistance Research Group and The Cancer Biology Center, Department of Pharmaceutical Sciences, \\ Texas Tech University Health Sciences Center, Amarillo, TX 79106, USA
}

Received July 12, 2007; Accepted August 16, 2007

\begin{abstract}
P-glycoprotein (Pgp), a plasma membrane (PM) glycoprotein, is responsible for the development of multidrug resistance. The mechanism by which Pgp is targeted to the $\mathrm{PM}$ is not defined. To identify proteins that influence Pgp trafficking, we utilized the yeast two-hybrid analysis procedure, which identified a new isoform of endoplasmic reticulum (ER)-bound Bap29, termed Bap29varP, as an interacting protein with the N-terminus of Pgp. The drugresistant human breast cancer MCF-7 (MCF-7/Adr ${ }^{\mathrm{R}}$ ) cells express both Bap29varP and $170 \mathrm{kDa}$ Pgp, which are however absent in the drug-sensitive MCF-7 cells. When Bap29varP was overexpressed in MCF-7/Adr ${ }^{\mathrm{R}}$ cells, Pgp was predominantly localized in the ER and intracellular vesicles, suggesting Bap29varP influences Pgp trafficking. When Pgp was expressed in MCF-7 cells, it was exclusively found in the ER with a molecular mass of $\sim 160 \mathrm{kDa}$ slightly smaller than that of the molecular mass of Pgp expressed in MCF-7/Adr ${ }^{\mathrm{R}}$ cells. On the other hand, when Pgp was expressed in Bap29varP-containing human colon adenocarcinoma HT-29 cells, it was localized at the PM. These findings together suggest that Bap29varP acts as an essential chaperone, influencing the processing and trafficking of Pgp to the cell surface.
\end{abstract}

Correspondence to: Dr U.S. Rao, Department of Pharmaceutical Sciences, School of Pharmacy, TTUHSC, Amarillo, TX 79106, USA

E-mail: us.rao@ttuhsc.edu

Abbreviations: MDR, multidrug resistance; Pgp, P-glycoprotein; $\mathrm{ABC}$, ATP binding cassette; CFTR, cystic fibrosis transmembrane conductance regulator; Bap29, B-cell antigen receptor-associated protein of $29 \mathrm{kDa}$; Bap29varP, Bap29 variant interacting with Pgp; Bap31, B-cell antigen receptor-associated protein of $31 \mathrm{kDa}$; PM, plasma membrane; ER, endoplasmic reticulum; TM, transmembrane; $\mathrm{ENaC}$, amiloride-sensitive epithelial $\mathrm{Na}+$ channel

Key words: cancer drug resistance, multidrug resistance, P-glycoprotein, yeast two-hybrid, Bap29, Bap31, protein trafficking

\section{Introduction}

Cancers undergoing chemotherapy often develop resistance to a wide variety of anticancer drugs and this phenomenon is known as multidrug resistance (MDR). While detoxifications of drugs, alterations in the cellular targets of anticancer drugs and pro-apoptotic pathways result in the development of drug resistance phenomenon, active anticancer drug efflux by the members of a large family of proteins commonly known as ABC-transporters has been well established as the most common mechanism of MDR in cancer cells (1). Pgp, a PMbound active drug-transporter and an important member of the above transporter family, is a 1280 amino acid long polypeptide, which folds into two distinct $\mathrm{NH}_{2}$-half and $\mathrm{COOH}$ half domains $(2,3)$. Each of these domains is $\sim 600$-amino acid long and contains six TM segments and an ATP binding domain. A unique and highly hydrophilic stretch of $\sim 75$ amino acids (residues 633-709), known as the linker region lies in between the two halves of Pgp $(4,5)$. The estimated molecular mass of Pgp is $\sim 140 \mathrm{kDa}$. Pgp contains three $\mathrm{N}$-glycosylation sites in the first extracellular loop and the extent of $\mathrm{N}$-glycosylation is dependent on the cell lines in which Pgp is expressed. For instance, Pgp expressed in breast cancer MCF-7/ $\mathrm{Adr}^{\mathrm{R}}$ cells is $\sim 170 \mathrm{kDa}$, whereas, it is $\sim 148$ $\mathrm{kDa}$ in Sf9 insect cells (6).

It has been well established that Pgp is a drug-translocating ATPase, which extrudes many anticancer drugs from the PM of cancer cells by utilizing the energy released from the ATP hydrolysis. While the drug-dependent ATPase catalytic cycle is well described $(7,8)$, the mechanism by which Pgp is trafficked to the PM is essentially unknown at present. Thus, it has been assumed that Pgp trafficks to the PM from its synthesis in the ER by following the classical anterograde pathway. While this assumption is reasonable as many PM-bound proteins invariably follow this trafficking route, it does not identify regulatory mechanisms, if any, that control the Pgp trafficking to the PM.

Interestingly, recent studies have identified two ERbound proteins termed Bap29 (9-12) and Bap31 (13-15) as regulators of the forward trafficking of several membrane proteins. These two ER proteins share $\sim 49 \%$ amino acid sequence homology and complete topology and form heterooligomeric structures in the ER. These proteins contain three TM segments with their N-termini exposed into the ERlumen and C-termini into the cytosol. It has been specifically 
shown that Bap31 binds to several membrane-bound proteins including immunoglobulin $\operatorname{IgD}(9,16)$, major histocompatibility Class I molecules $(11,17)$, tetraspanins (18), CFTR (19), cytochrome 450 (20) and B2-integrins (18). Importantly, such Bap31-membrane protein interactions are shown to regulate the ER-retention and -exit of the above membrane proteins, implying that the Bap31/29 act as molecular chaperones involved in the membrane protein trafficking.

Studies have shown that the linker region in the Pgp molecule plays a critical role in the drug efflux and ATPase functions of $\operatorname{Pgp}(5,21)$, and is well accessible to interactions with different proteases $(22,23)$, indicating that this exposed region is likely to be a site for protein-protein interactions in vivo. We have recently reported that $\mathrm{RNF} 2$, an E3 ubiquitin ligase interacts with this region of the molecule and regulates the Pgp ATPase function (24). To further identify proteins that interact with other regions of Pgp, here we used the $\mathrm{NH}_{2}$-terminus of Pgp as bait and screened the prostate adenocarcinoma cDNA library by the yeast twohybrid procedure. This analysis identified a new and highly homologous isoform of Bap29, termed here Bap29varP, as an interacting protein. Since Bap29 is involved in the membrane protein trafficking, the isolation of Bap29varP as an interactor raised a distinct possibility that this protein regulates Pgp trafficking to the cell surface. We investigated this possibility in detail and the results presented in this report show that Bap29varP interacts with Pgp in the ER and such interactions influence the proper post-translational modifications and the subsequent forward trafficking of Pgp to the cell surface.

\section{Materials and methods}

Materials. Alexa Fluor-coupled antibodies and anti-hexahistidine antibodies were obtained from Invitrogen, CA. SDSPAGE and Western blotting reagents were purchased from Bio-Rad, CA. All other reagents used were of analytical grade.

Molecular biological methods. Standard recombinant DNA procedures of plasmid DNA preparation and subcloning into vectors were used.

Yeast two-hybrid screening. The pGBKT7 and pGADT7 vectors, yeast strains AH109 and Y187 and the procedures of yeast two-hybrid analysis were derived from the Matchmaker Two-Hybrid System 3 (Clontech, CA) and the screening was carried out essentially as described previously (24). Briefly, the human prostate adenocarcinoma cDNA library subcloned into pGADT7 vector was transformed into AH109 to generate the prey. The cDNA encoding NT-Pgp (the 1-50 amino acid stretch from the N-terminus), LR-Pgp (amino acids 633-707 representing the linker region), CT-Pgp (amino acids 12021280 located at the C-terminus) and NT- $\alpha \mathrm{ENaC}$ (1-40 amino acids of the $\alpha$-subunit of the amiloride-sensitive epithelial sodium channel, $\alpha E N a C$ ) were amplified by PCR from the $A B C B 1$ and $\alpha E N \alpha C$ cDNAs, subcloned into pGBKT7 vector and then transformed into the yeast Y187 strain to generate the bait strains. The pGBKT7/NT-Pgp containing bait strain was mated with the AH109 prey. The diploid yeast colonies resulting from this mating exhibited both growth on quadruple dropout (trp-, leu-, ade- and his-) SD minimal agar medium and $\beta$-galactosidase activity on X- $\alpha$-Gal (5-bromo-4chloro-3-inoyl- $\alpha$-D-galactopyranoside). The pGADT7 library plasmids from the positive clones were isolated and sequenced at The University of Nebraska DNA Sequencing Facility, Lincoln, NE. The insert DNA sequences were identified using the NCBI sequence database using the BLAST network service.

Mammalian two-hybrid assay. To determine the proteinprotein interactions, the mammalian 2-hybrid assay was carried using the Mammalian Matchmaker Two-Hybrid Assay kit (Clontech). The GAL4 DNA-binding domain cloning vector $\mathrm{pM}$, the activation-domain cloning vector pVP16, and the secreted alkaline phosphatase reporter construct pG5SEAP were parts of the kit. The Bap29varP cDNA in the pGADT7 vector was subcloned into pVP16 and generated pVP16/Bap29varP construct. To prepare the $\mathrm{pM} / \mathrm{NT}-\mathrm{Pgp}, \mathrm{pM} / \mathrm{CT}-\mathrm{Pgp}, \mathrm{pM} / \mathrm{LR}-\mathrm{Pgp}$ and $\mathrm{pM} / \mathrm{NT}-\alpha \mathrm{ENaC}$ constructs, cDNAs coding for the NT-Pgp, CT-Pgp and LR$\mathrm{Pgp}$, described above were subcloned into the $\mathrm{pM}$ vectors, respectively. HEK293 cells plated at an initial density of $6 \times 10^{5}$ cells per well in a 6 -well plate were transfected with pG5SEAP $(0.1 \mu \mathrm{g})$ and specific combinations of $\mathrm{pM}, \mathrm{pM} /$ NT-Pgp, pM/CT-Pgp and pM/LR-Pgp vector (1.0 $\mu \mathrm{g}$ each) and pVP16/Bap29varP vector $(1.0 \mu \mathrm{g})$, using the calcium phosphate transfection procedure. The medium collected at $48 \mathrm{~h}$ post-transfection was analyzed for the presence of alkaline phosphatase activity by measuring the chemiluminescence resulting from the hydrolysis of disodium 3(4-methoxys piro[1,2-diox etane-3,2'-(5'-chloro)tricyclo [3.3.1.1(3,7)]decan]-4-yl) phenyl phosphate (CSPD). These experiments were repeated three times, with each experiment carried out in triplicate wells.

Cell lines, culture conditions and transfections. MCF-7/Adr ${ }^{\mathrm{R}}$ (25), HEK-293 and HT-29 (purchased from ATCC) cells were maintained in DMEM medium. The drug-sensitive human breast cancer MCF-7 (purchased from ATCC, Cat. No. HTB-22) cells were maintained in Earl's Minimal Medium supplemented with insulin. The Chinese hamster ovary $(\mathrm{CHO})$ cells were grown in F-12 medium. The growth media were supplemented with $10 \%$ (v/v) FBS and antibiotics (penicillin, 100 units $/ \mathrm{ml}$ and streptomycin, $100 \mu \mathrm{g} / \mathrm{ml}$ ) and grown in a $37^{\circ} \mathrm{C}$ humidified incubator with $5 \% \mathrm{CO}_{2}$. To express recombinant proteins, approximately $3.5 \times 10^{5}$ cells were seeded in each well of 6-well plates with or without coverslips and transfected with different plasmids using Lipofectamine or Lipofectamine-2000 reagent (Invitrogen) as recommended by the manufacturer. Cells were analyzed $24 \mathrm{~h}$ post transfection by immunocytochemistry and Western blotting.

Bap29varP cDNA. Isolation of total RNA, reverse transcription and PCR were described previously (26). The number of PCR cycles was 30 . The $0.9 \mathrm{~kb}$ Bap29varP cDNA was isolated from the total RNA of MCF-7 cells using the Bap29varP-specific forward primer, 5'-ATGACACTCC AATGGGCTGCAG-3', and reverse primer 5'-ACAATAA 
ATTTATTCCCATAGTTCTGGAGGCA-3'. Restriction sites, BglII site and $\mathrm{KpnI}$ were added to these primers to facilitate subcloning. To express a GFP-fused Bap29varP (GFP-Bap29varP), the Bap29varP cDNA was subcloned in-frame at the 3'-end of GFP cDNA in the pEGFP-C1 vector. Bap29varP thus expressed was termed GFP-Bap29varP. Similarly, a cDNA encoding HA-epitope was added to the 5'- of the Bap29varP cDNA to express HA-Bap29varP.

Immunofluorescence and confocal imaging. Immunocytochemistry was carried as described (26). Briefly, cells on coverslips transfected with plasmids were fixed with paraformaldehyde to stain with anti-Pgp antibody UIC2 (27). Cells were also fixed with acetone:methanol (1:1) and then NP-40 permeabilized to immunostain with anti-Pgp NH211, antiGFP, anti-HA, anti-calreticulin, anti-GM130 and anti-EEA1. Cells were then incubated with AlexaFluor-488 or -594coupled anti-rabbit or -mouse antibodies (Molecular Probes, Eugene, OR) depending on the type of primary antibody used in the study. Coverslips were mounted with Antifade (Molecular Probes) and then analyzed on a Leica laser confocal microscope (TCS SL; Leica Microsystems, Heidelberg, $\mathrm{GmbH}$ ), with $63 \mathrm{X} / 1.4$ oil objective and immersion oil. GFP and AlexaFluor-488 were excited at $488 \mathrm{~nm}$ and detected through a $505-530 \mathrm{~nm}$ bandpass filter. AlexaFluor-594 was excited at $543 \mathrm{~nm}$ and detected through a $615 \mathrm{~nm}$ bandpass filter.

SDS-PAGE and Western blotting. SDS-PAGE and Western blotting were carried as described previously (26).

Antibodies. Anti-Bap29varP was raised to the amino acid sequence in Bap29varP, 249-AEIGVMEPQQRNADSH QKLEEAKN-272 in rabbit at a commercial location (AnimalPharm Services, CA). Immunoblotting was used to screen for the presence of antibody. Characterization of anti-Pgp $\mathrm{NH}_{2} 11$ was described previously (26). The anti-Pgp C219 was purchased from Calbiochem, CA and UIC2 and anti-GFP were from Chemicon, Temecula, CA. The anticalreticulin, anti-GM130, anti-EEA1 antibodies were purchased from Affinity Bioreagents, Golden, CO.

Protein estimation. The protein content in the samples was estimated by the BioRad's DC Protein Assay kit using bovine serum albumin as standard.

\section{Results}

Identification of Bap29varP as an interacting protein of Pgp. To identify proteins that directly interact with Pgp, we performed a yeast two-hybrid screen of a human prostate adenocarcinoma cDNA library using the N-terminal 1-50 amino acid stretch of Pgp (NT-Pgp) as bait, as described in Materials and methods. Several diploid yeast colonies resulting from this mating were positive for histidine biosynthesis and B-galactosidase activity. DNA sequence analysis of the positive clones revealed the presence of portions of several known gene products including translocon subunit as well as DNA fragments whose sequences are yet to be defined. However, a few diploid yeast clones

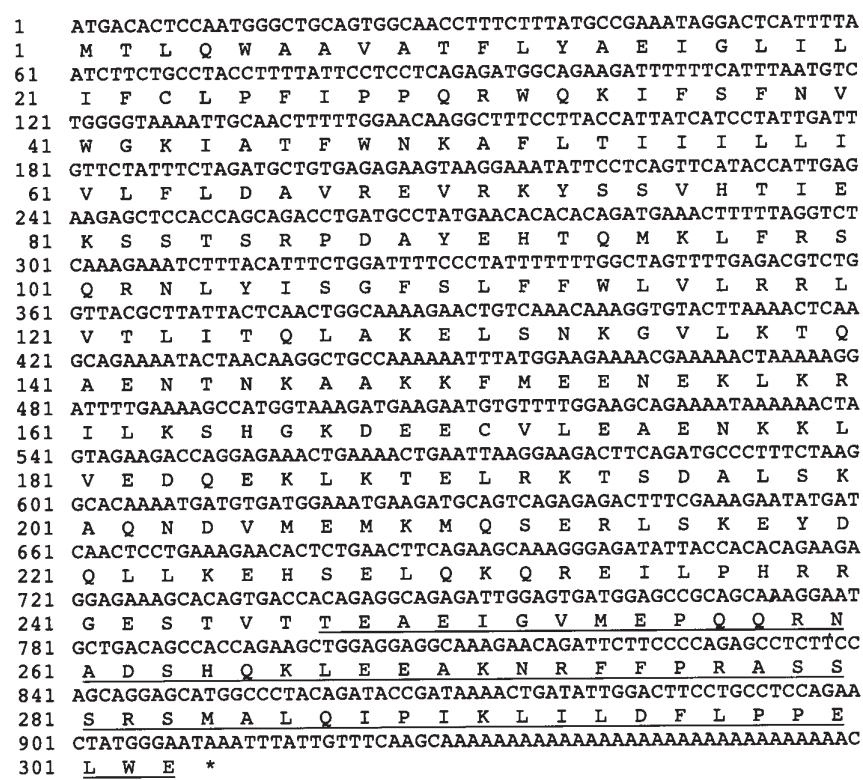

Figure 1. Coding sequence of Bap29varP. The deduced cDNA and amino acid sequence of Bap29varP isolated from MCF-7 cell line is shown here. The underlined sequence is the interacting peptide encoded by the DNA sequence present in the pGADT7/Bap29varP clone, which interacts with the N-terminus of Pgp. The 3'-end sequence of the cDNA in the pGADT7/ Bap29varP is also presented.

contained an identical cDNA insert encoding a 58 amino acid long polypeptide, stop codon followed by poly A tail (Fig. 1, underlined). Homology search in the NCBI database revealed that this amino acid sequence was $\sim 93 \%$ homologous to the C-terminal sequence in Bap29 isoform 3 (NM_001008406) (Fig. 2). Further BLAST analysis of NCBI database indicated that Bap29 isoform 3 is highly homologous to three additional proteins, which are Bap29 (isoform 2) $(9,16)$, Bap29 isoforms 1 and 4. Interestingly, all of these isoforms share complete amino acid sequence from their $\mathrm{N}$-termini, but differ at their C-termini due to different lengths (Fig. 2), perhaps due to alternative splicing. Because of the homologous nature of the above isolated clone to Bap29 proteins and its interaction with Pgp, the full-length clone of the interacting protein is termed Bap29varP, as it is a Bap29 variant interacting with Pgp.

To confirm the interaction between the 58 amino acid C-terminal stretch of Bap29varP and NT-Pgp, different yeast baits carrying NT-Pgp, CT-Pgp and NT- $\alpha \mathrm{ENaC}$ were mated with the above Bap29varP fragment present in the pGADT7/Bap29varP prey vector. Fig. 3A shows that only the diploids resulting from mating between the NT-Pgp and Bap29varP were able to grow on the quadruple drop-out SD agar media and hydrolyzed $\mathrm{X}-\alpha-\mathrm{Gal}$, suggesting that the interaction between the C-terminus of Bap29varP and $\mathrm{N}$-terminus of Pgp is specific.

To determine if the 58 amino acid C-terminal stretch of Bap29varP interacts with different region of Pgp in mammalian cells, the mammalian two-hybrid assay in HEK293 cells was carried out as described in Materials and methods. The strength of protein-protein interactions was measured as the amount of alkaline phosphatase activity in the into the culture medium. Fig. 3B shows that cells 


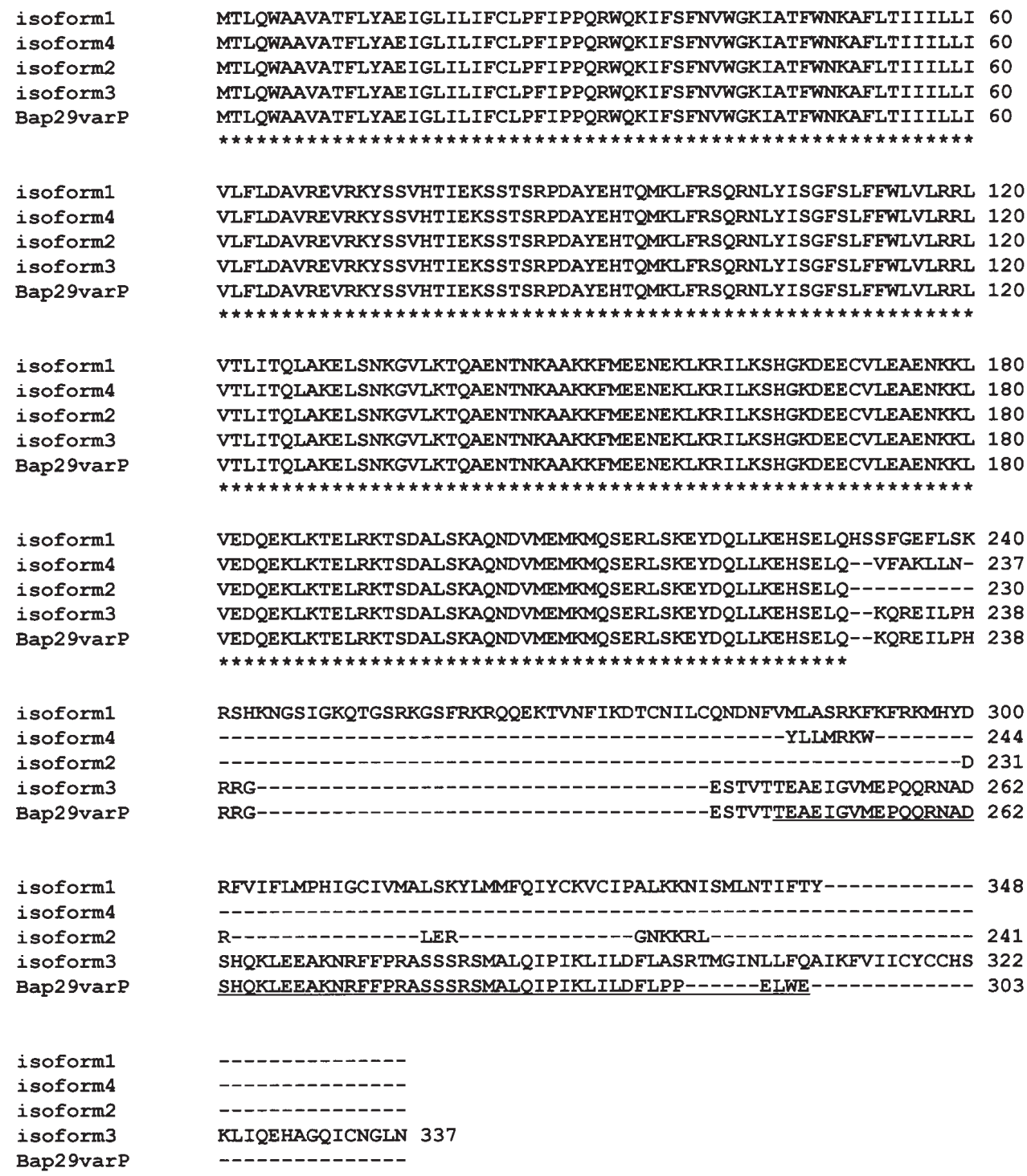

Figure 2. Alignment of Bap29varP sequence with different Bap29 isoforms. Full-length amino acid sequences of different Bap29 isoforms identified in the NCBI database were aligned with Bap29varP sequence using the web-based CustalW program. The asterisks below each cluster of amino acids indicate complete homology of the amino acid in all proteins.

transfected with Bap29varP and NT-Pgp cDNAs secreted significantly higher amount of alkaline phosphatase into the medium, when compared to other combinations of transfections, suggesting that the N-terminus of Pgp is a strong interacting domain with the C-terminus of Bap29varP.

It can be seen in Fig. 2 that Bap29 isoform 3 contains an identical Pgp-interacting sequence at its $\mathrm{C}$-terminus followed by an extra 34 amino acid stretch. To test if these extra 34 residues influence the interactions with Pgp, the C-terminal 91 amino acid stretch of Bap29 isoform 3 that includes the Pgp-interacting sequence was tested for its ability to interact with different regions of Pgp by yeast two-hybrid assay. Results of this study indicated that this C-terminus of Bap29 isoform 3 does not interact with any of the regions of Pgp (data not shown), suggesting that the presence of additional 34 amino acid stretch $\mathrm{C}$-terminus to the Pgp-interacting sequence prevents interactions with Pgp. We also tested the possibility that the C-terminal 74 amino acid stretch of Bap31, a protein that shares $\sim 49 \%$ sequence homology with Bap29varP, interacts with different regions of Pgp by the above mentioned yeast hybrid assay. Results of this study indicated that the C-terminus of Bap31 does not interact with any of the regions of Pgp (data not shown). Taken together, these experiments suggest that Bap29varP is a specific interacting protein that interacts with Pgp.

Full-length Bap29varP transcript is found in different cell lines. Since the Pgp interacting peptide sequence is conserved in Bap29 isoform 3 (Fig. 2), we reasoned that these two proteins share complete homology at their identical $\mathrm{N}$-termini. To confirm this possibility, a forward primer encompassing the start codon of Bap29 isoform 3 and a reverse primer encompassing the stop codon of Bap29varP were used in a PCR using reverse transcript mixtures prepared from different cell lines as templates, as described in Materials and methods. Theoretically, a single $0.9 \mathrm{~kb}$ cDNA would be amplified if the predicted Bap29varP cDNA is transcribed in the cells. As predicted, Fig. $3 \mathrm{C}$ shows the amplification of a single $0.9 \mathrm{~kb}$ DNA fragment in both the drug-sensitive MCF-7 and HT-29 cells and drug-resistant MCF-7/Adr ${ }^{\mathrm{R}}$ 
A
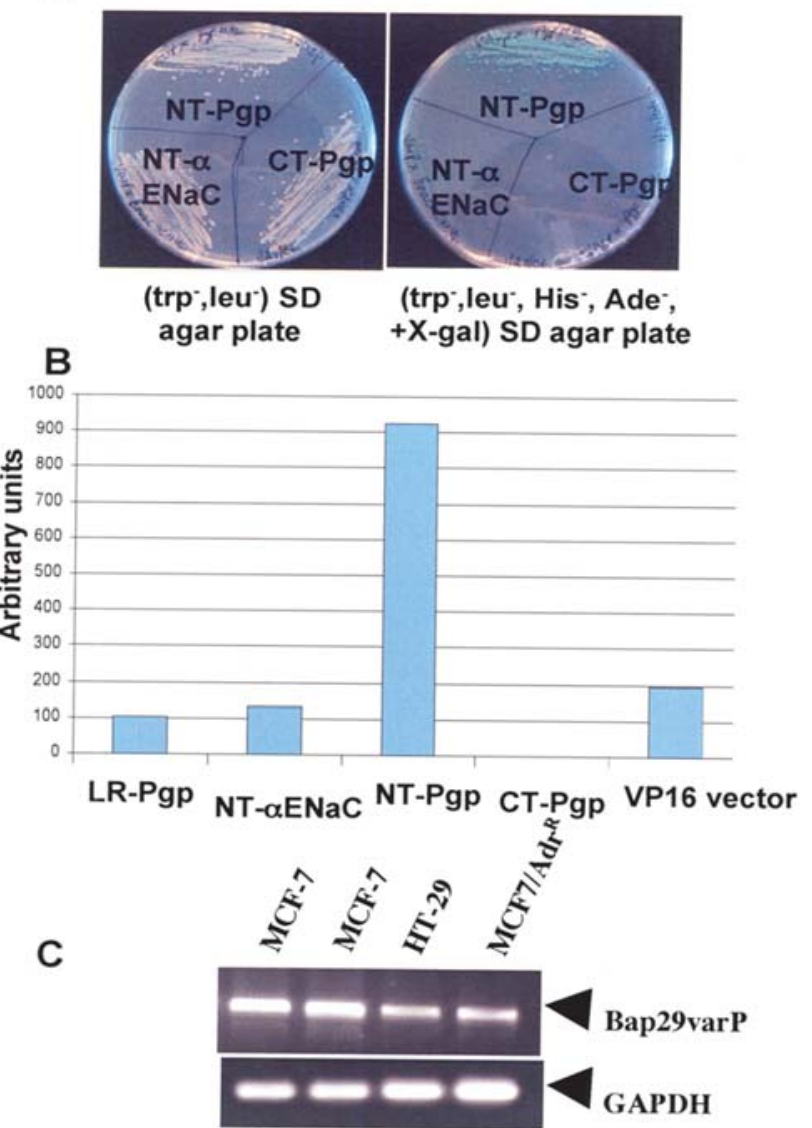

D

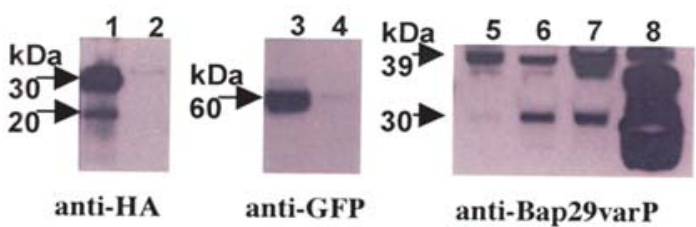

Figure 3. Yeast two-hybrid analysis. (A) Yeast diploids expressing either NT-Pgp, CT-Pgp or NT- $\alpha$ ENaC and Bap29varP peptide were plated on double (left) and quadruple (right) SD agar plates containing $\alpha$-X-gal as described in Materials and methods. Growth of blue colonies in quadruple dropout plate indicates interaction between bait and prey proteins. (B) Analysis of interaction between Bap29varP and Pgp by mammalian two hybrid assay. Mammalian two hybrid assay was carried out in HEK-293 cells as described in Materials and methods. Briefly, the cells were cotransfected with vectors carrying sequences of Bap29varP and different regions of Pgp and $\alpha \mathrm{ENaC}$ and secreted alkaline phosphatase. As a control, VP16 vector was used. Secreted alkaline phosphatase was assayed by measuring the chemilumenscence of CRPD substrate hydrolysis as described in Materials and methods. The enzymatic activity was expressed as arbitrary units. (C) RT-PCR analysis of Bap29varP expression. Equal amounts of cDNAs obtained from $10 \mu \mathrm{g}$ of total RNA isolated from MCF-7, HT-29 and MCF-7/Adr ${ }^{\mathrm{R}}$ cells were used in the PCR using Bap29varP-specific primer set as described in Materials and methods. Glyceraldehyde-3-phosphate dehydrogenase (GAPDH) was amplified to serve as an internal control. The expected $\sim 0.9 \mathrm{~kb}$ Bap29varP cDNA is amplified in all of the cell lines, which is indicated with an arrow. (D) Western blot analysis of Bap29varP expression. $\mathrm{CHO}$ cells were transfected with HA-epitope tagged-(lanes 1 and 8) and GFP-tagged-(lane 3) Bap29varP and control plasmid (lanes 2 and 4 ) for $24 \mathrm{~h}$. The cells were collected and the protein was precipitated with 6\% trichoroloacetic acid and then dissolved in Laemmli disaggregating buffer. Protein $(10 \mu \mathrm{g})$ from each sample was loaded on SDS-PAGE followed by electroblotting onto PVDF membrane, as described (26). The blots were developed using anti-HA and anti-GFP, respectively. MCF-7 (lane 5), MCF-7/ $\mathrm{Adr}^{\mathrm{R}}$ (lane 6) and HT-29 (lane 7) cell lysates were analyzed by Western blotting using anti-Bap29varP. The $\sim 30 \mathrm{kDa}$ protein in MCF-7/Adr ${ }^{\mathrm{R}}$ (lane 6) and HT-29 (lane 7) cells is the endogenous Bap29varP. The $\sim 39 \mathrm{kDa}$ protein is predicted as Bap29 isoform 3. cells. Although the amount of amplified cDNA from these was slightly variable in different experiments, it is suffice to indicate that all these cells contain Bap29varP transcript. DNA sequencing of the fragment amplified from MCF-7 cells was in complete agreement with the sequence shown in Fig. 1, indicating that Bap29varP is a new isoform of Bap29 and is constitutively transcribed in many cancer cells. The open reading frame of this cDNA contains a 909 bp encoding 303 amino acid protein predicted in Fig. 1. Predictive algorithms suggest that Bap29varP is an ER protein containing three TM segments, with its $\mathrm{N}$ - and $\mathrm{C}$-termini located in the ER-lumen and cytosol, respectively, similar to the topology of Bap29 and Bap31.

Expression of Bap29varP. To detect Bap29varP, HA- and GFP-epitopes encoding cDNA segments were added to the 5 '-end of the Bap29varP cDNA, expressed in CHO cells and then analyzed by Western blotting, as described in Materials and methods. Fig. 3D shows that cells transfected with the HA-Bap29varP cDNA expressed an anti-HA antibody reactive $\sim 30 \mathrm{kDa}$ protein, which is slightly smaller than the expected $35 \mathrm{kDa}$ for Bap29varP. Control plasmid transfected cells did not have any antibody-reactive proteins. Similarly, CHO cells transfected with GFP-Bap29varP cDNA expressed an anti-GFP reactive $\sim 60 \mathrm{kDa}$ protein, which is similar to the expected size of $\sim 65 \mathrm{kDa}$ for GFP-Bap29varP. Because the control cell lysates did not contain any antibody reactive proteins, it is reasonable to suggest that transfection of cells with the HA-Bap29varP cDNA results in the expression of full length Bap29varP, which migrates as $30 \mathrm{kDa}$ protein in SDS-PAGE.

Raising an antibody that selectively recognizes Bap29varP is hampered by the fact Bap29varP is highly homologous to Bap29 isoform 3 (Fig. 2). Since these two proteins are highly homologous but different in their expected molecular masses (the molecular mass of Bap29 isoform 3 is $\sim 39 \mathrm{kDa}$ ), we raised an antibody to the epitope 249-AEIGVMEPQQRN ADSHQKLEEAKN-272 in Bap29varP, which is expected to recognize both Bap29varP and Bap29 isoform 3 in Western blots. Although the anti-Bap29varP prepared for this study binds to both of these proteins, which can however be distinguished by Western blotting due to their differential migration on SDS-PAGE. Thus, anti-Bap29varP prepared as described in Materials and methods, was used to analyze the expression of Bap29varP and Bap29 isoform 3 in different cancer cells by Western blotting and the results are shown in Fig. 3D. An $~ 39-k D a$ protein immunoreactive with antiBap29varP is present in both MCF-7, HT-29 and MCF-7/ $\mathrm{Adr}^{\mathrm{R}}$ cells, which is likely the Bap29 isoform 3. An $\sim 30 \mathrm{kDa}$ protein uniquely present in both HT-29 (lane 7) and MCF-7/ $\operatorname{Adr}^{\mathrm{R}}$ (lane 6) cells in high levels, which was nearly absent in MCF-7 cells (lane 5). The HA-Bap29varP expressed in $\mathrm{CHO}$ cells was resolved into several proteins in the $20-40 \mathrm{kDa}$ range (lane 8), which could be due to degradation and aggregation. Although not shown, the immunostaining of these proteins was completely abolished when the antiBap29varP was pre-incubated with immunizing peptide. These results together suggest that Bap29varP is expressed in both HT-29 and MCF-7/Adr ${ }^{\mathrm{R}}$ but not in the drug-sensitive MCF-7 cells. 
A. MCF-7

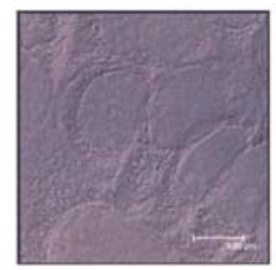

B. MCF-7/Adr ${ }^{R}$

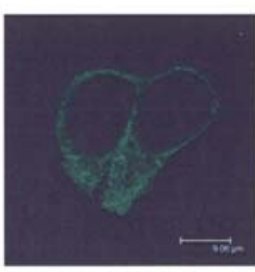

GFP-Bap29varP

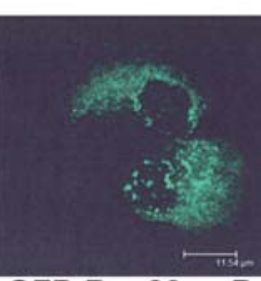

GFP-Bap29varP

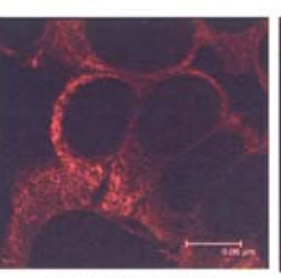

calreticulin

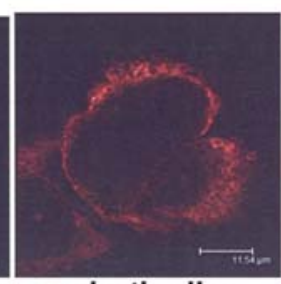

calreticulin overlay
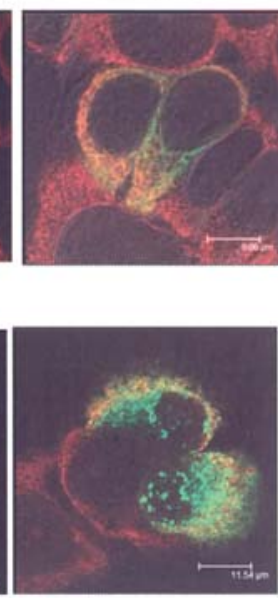

Figure 4. Analysis of Bap29varP expression by confocal laser microscopy. GFP-Bap29varP was transiently expressed in MCF-7 (A) and MCF-7/Adr ${ }^{\mathrm{R}}$ cells (B) for $24 \mathrm{~h}$. The cells were fixed and then stained with anti-calreticulin (panel: calreticulin) followed by Alexafluor-594 coupled secondary antibody. The green florescence of GFP-Bap29varP and Alexafluor-594 associated calreticulin were visualized by confocal laser microscopy and the images were superimposed (right side panel). The yellow color indicates the co-localization of Bap29varP and calreticulin.

A

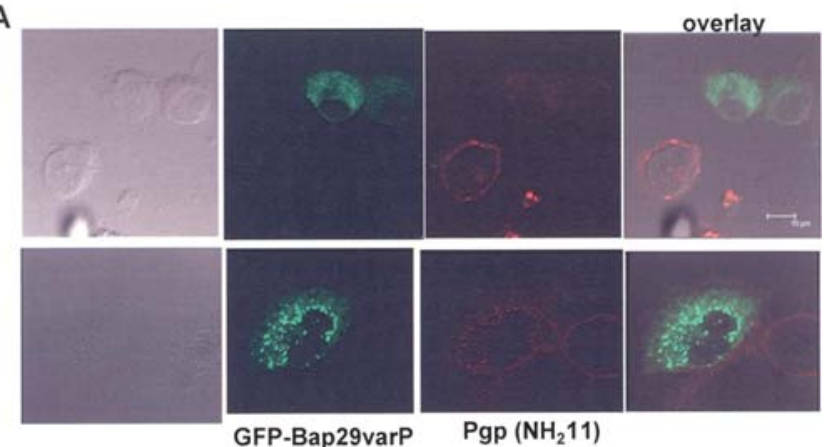

B

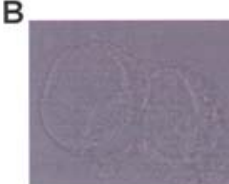

C

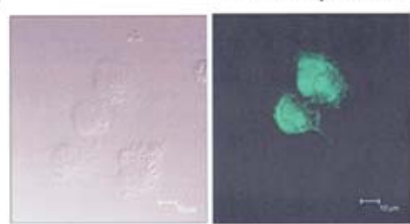

GFP

Pgp (UIC2)
A
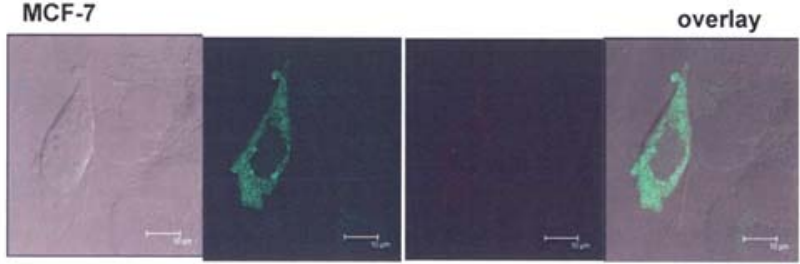

B

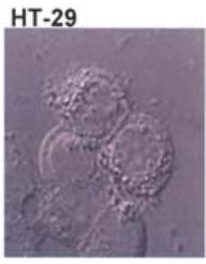

HA-Pgp(anti-HA) Pgp (UIC2)

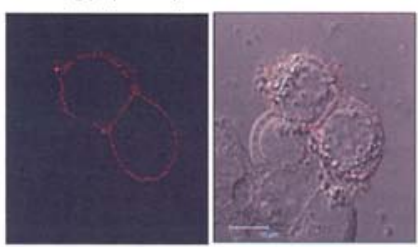

Pgp (UIC2)

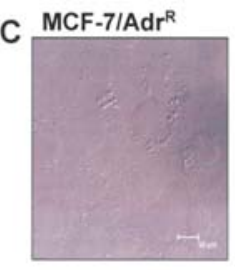

Figure 5. Bap29varP co-localizes with Pgp. MCF-7/Adr ${ }^{\mathrm{R}}$ cells were transiently transfected with GFP-Bap29varP cDNA for $24 \mathrm{~h}$. The cells were fixed and stained with Pgp-specific NH211 (A) and UIC2 (B) in conjunction with AlexaFluor-594-coupled secondary antibodies. The fluorescence associated with Bap29varP and Pgp were visualized by confocal laser microscopy. Images are superimposed digitally (right side panel). (C) MCF-7/ $\mathrm{Adr}^{\mathrm{R}}$ cells were transfected with GFP vector were used as control. The subcellular location of Pgp was determined using UIC2 antibody as described.

Bap29varP is an ER protein. To determine the subcellular distribution of Bap29varP, GFP-Bap29varP was expressed in MCF-7 and MCF-7/Adr ${ }^{\mathrm{R}}$ cells and immunostained for different subcellular marker proteins, and then analyzed by confocal laser microscopy. Fig. 4A shows that the GFP fluo- rescence associated with GFP-Bap29varP was uniformly distributed in the intracellular membranous regions of MCF-7 cells, excluding the nuclear region. Although the distribution of Bap29varP in MCF-7/Adr ${ }^{\mathrm{R}}$ cells is similar, Bap29varP was also found in distinct vesicular structures in the cytosolic 
region. To further define the cellular location of this protein, the transfected cells were also immunostained for different subcellular marker proteins including antibodies against calreticulin, an ER protein; GM130, a Golgi-bound protein; and EEA1, an early endosomal protein and then analyzed by confocal laser microscopy. Results of these experiments indicated that GFP fluorescence of GFP-Bap29varP is colocalized only with calreticulin in both MCF-7 (Fig. 4A) and $\mathrm{MCF}-7 / \mathrm{Adr}^{\mathrm{R}}$ cells (Fig. 4B), but not with Golgi or early endosomes (not shown), suggesting that Bap29varP is an ER protein. These results also showed that the vesicular structures in which GFP-Bap29varP was present in MCF-7/Adr ${ }^{\mathrm{R}}$ cells do not appear to contain sufficient amounts of calreticulin to suggest that these are non-ER-vesicles. These results together suggested Bap29varP is an ER protein, which accumulates into vesicular structures in drug-resistant $\mathrm{MCF}-7 / \mathrm{Adr}^{\mathrm{R}}$ cells.

Bap29varP co-localizes with Pgp in the ER of MCF-7/Adr ${ }^{R}$ cells. To determine if Bap29varP interacts with Pgp, $\mathrm{MCF}-7 / \mathrm{Adr}^{\mathrm{R}}$ cells were transfected with the GFP-Bap29varP cDNA, fixed with acetone:methanol mixture, permeabilized with NP-40 and then stained with anti-GFP and $\mathrm{NH}_{2} 11$ in conjunction with AlexaFlor-488 and -594-coupled secondary antibodies, respectively, as described in Materials and methods. The cells were stained with anti-GFP as the GFP fluorescence is quenched due to fixing of cells with acetonemethanol solvent. The cells were analyzed by confocal laser microscopy and the results are shown in Fig. 5. As expected from Fig. 4A, Bap29varP was localized both in the ER and intracellular vesicles. Pgp in cells expressing GFP-Bap29varP was present both in the ER and intracellular vesicles (Fig. 4A), which is superimposable with the distribution of Bap29varP. However, the amount of Pgp at the PM of cells that were transfected with Bap29varP was greatly reduced. To further confirm the reduction of Pgp levels at the PM due to Bap29varP expression, MCF-7/Adr ${ }^{R}$ cells transfected with GFP-Bap29varP cDNA were fixed in paraformaldehyde without permeabilization and then stained with Pgp-specific UIC2. This staining procedure did not affect the GFP fluorescence. UIC2 antibody has been shown previously to immunostain Pgp only from the extracellular side of the molecule (27), suggesting that this antibody identifies Pgp at the PM. Fig. 5B shows that cells expressing GFPBap29varP contained reduced amount of Pgp at the PM, when compared to the amount of Pgp in the untransfected cells. It may be noted that Bap29varP was not detectable at the PM in any of our experiments. Finally, cells transfected with GFP vector plasmid did not alter the expression of Pgp at the PM (Fig. 5C). Taken together, these results suggested that Bap29varP interacts with Pgp in the ER, resulting in the accumulation of Pgp in the ER and intracellular vesicles, with concomitant reduction of Pgp levels at the PM.

Bap29varP is necessary for the cell surface expression of Pgp. To determine the functional role of Bap29varP in the reduction of Pgp levels at the PM of $\mathrm{MCF}-7 / \mathrm{Adr}^{\mathrm{R}}$ cells, an HA-epitope tag was added to the N-terminus of Pgp using standard molecular biology methods, and then expressed in
Bap29varP- MCF-7 and Bap29varP' but Pgp- HT-29 cells and its subcellular distribution was determined by confocal laser microscopy. The HA-epitope was added to Pgp to detect the expressed Pgp selectively using anti-HA antibody. The transfected cells were fixed and then immuno-stained using anti-HA (rabbit polyclonal) and UIC2 (mouse monoclonal) as primary antibodies in conjunction with AlexaFluor-488 and -594 coupled secondary antibodies, respectively. Fig. 6A shows the confocal laser microscopy analysis of the intracellular and the PM-bound Pgp in these cells. Pgp expressed in MCF-7 cells was intracellular, and more specifically co-localized with calreticulin (data not shown), which was not immunostained with UIC2 (Fig. 6A), suggesting that Pgp did not reach the PM in these cells. Loo and Clarke have previously observed that mutant Pgp forms that fail to reach the cell surface in HEK-293 cells could reach the PM upon treatment with the Pgp substrates including cyclosporine A, vinblastine and verapamil, suggesting that Pgp substrates act as molecular chaperones for Pgp for forward trafficking (28). Since Pgp expressed in MCF-7 cells does not reach the PM, we thus tested if cyclosporine A could facilitate the forward trafficking of Pgp to the cell surface. HA-epitope tagged Pgp was expressed in MCF-7 cells for $24 \mathrm{~h}$ and then incubated with 0,5 and $10 \mu \mathrm{M}$ cyclosporine A for $6 \mathrm{~h}$. The cellular location of Pgp in these cells was analyzed by confocal laser microscopy as described above. However, cyclosporine A did not show any effect on the forward trafficking of Pgp to the PM (data not shown). Although it is not clear for the lack of cyclosporine A effects on the forward trafficking of Pgp, it is likely that the differences in the nature of MCF-7 and HEK-293 cells are responsible for this phenomenon. In addition, our observations are based on direct visualization of Pgp at the cell surface using confocal laser microscopy, whereas, the study of Loo and Clarke employed the cell surface labeling and detection of $\sim 170 \mathrm{kDa}$ Pgp (28).

Thus, the above results suggested that the expression of Bap29varP is necessary for Pgp to exit the ER and traffic to the PM. To test this possibility, we expressed the HA-epitope tagged Pgp in Bap29varP $\mathrm{P}^{+}$HT-29 cells, which do not express Pgp (data not shown) and the cellular location of expressed Pgp was determined. Fig. 6B shows that few cells were immunostained with UIC2, suggesting that these cells were transfected with Pgp cDNA and the expressed Pgp reached the PM. However, the anti-HA staining was extremely weak, probably due to the HA-epitope in the expressed Pgp is not accessible to the antibody, as these cells were fixed under non-permeabilizing conditions. As a control, MCF-7/ $\mathrm{Adr}^{\mathrm{R}}$ cells were uniformly immunostained with UIC2, which indicates the PM location of Pgp (Fig. 6C). Thus, these results suggest that Pgp trafficks to the PM in cells (HT-29 and $\mathrm{MCF}-7 / \mathrm{Adr}^{\mathrm{R}}$ ) constitutively expressing Bap29varP. Conversely, Pgp fails to reach the PM in cells that do not express Bap29varP protein.

Pgp expressed in Bap29varP- MCF-7 cells is post-translationally under modified. To further understand the above results that Pgp does not reach the PM in the absence of Bap29varP, the HA-epitope tagged Pgp was expressed in MCF-7 cells and then analyzed by Western blotting using the 


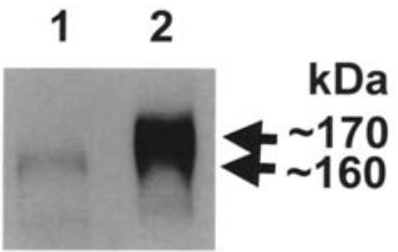

Figure 7. Western blotting analysis of Pgp expressed in Bap29varP -ve MCF-7 cells. MCF-7 (lane 1) and MCF-7/Adr ${ }^{\mathrm{R}}$ (lane 2) cells were transiently transfected with HA-tagged Pgp cDNA and vector control for $24 \mathrm{~h}$. The cells were lysed and $10 \mu \mathrm{g}$ protein from each cell lysate was separated on $7.5 \%$ SDS-PAGE and then transferred onto PVDF membranes. The immunoblots were developed using C219 antibody. Pgp expressed in MCF-7 cells is $\sim 160 \mathrm{kDa}$ and Pgp expressed in MCF-7/Adr ${ }^{\mathrm{R}}$ cells is $\sim 170 \mathrm{kDa}$

Pgp-specific C219 antibody. Fig. 7 shows that Pgp expressed in MCF-7 cells migrated as $\sim 160 \mathrm{kDa}$ protein (lane 1), which is slightly smaller than the $\sim 170 \mathrm{kDa}$ Pgp constitutively expressed in MCF-7/Adr ${ }^{R}$ cells (lane 2). Pgp expressed in Sf9 insect cells is non-glycosylated and migrated as $\sim 140 \mathrm{kDa}$ protein in these gels (data not shown). Due to low efficiency of transfection, Pgp expressed in HT-29 cells could not be detected by Western blotting. Since Pgp expressed in MCF-7 cells is present in the ER and migrates as a smaller protein than Pgp expressed in MCF-7/Adr ${ }^{R}$ cells, these results suggest that complete post-translational modification of Pgp is necessary for the forward trafficking to the cell surface. This interpretation is in agreement with the observations of Loo and Clarke (28), Wagenaar and co-workers (29) and Ambudkar and co-workers (30), who collectively demonstrated that N-glycosylation is necessary for the cell surface expression of Pgp. Thus, these observations together suggest that Bap29varP plays a specific chaperone role in the posttranslational modification of Pgp, which is necessary for its forward trafficking to the cell surface in MCF-7 cells.

\section{Discussion}

Pgp is a prominent PM-bound $\mathrm{ABC}$ transporter that mediates drug efflux from many human MDR cancers. Thus, the presence of Pgp at the PM is responsible for the drug resistance and chemotherapy failure. Therefore, it is reasonable that efforts in lowering cell surface expression of Pgp by blocking its forward trafficking from ER will likely facilitate successful cancer chemotherapy. While the regulatory mechanisms of $A B C B 1$ gene expression (reviewed in ref. 31), the in vitro biosynthesis and folding (32-34) and biochemical and catalytic aspects of $\operatorname{Pgp}(21,35,36)$ were investigated previously, the mechanism by which this drug transporter reaches the cell surface is essentially unknown. Much attention to the mechanism of bio-synthesis and trafficking has been directed to the wild-type CFTR, another plasma membrane-bound $\mathrm{ABC}$ transporter family member and a closely related protein to $\mathrm{Pgp}$, and its mutant CFTR 4508, largely owing to the fact that the latter fails to exit ER $(37,38)$.

To identify proteins that interact with Pgp, we have adapted the yeast two-hybrid analysis, which is widely used to determine protein-protein interactions. Recently, we used the linker region of Pgp as bait and identified RNF2 as an interacting protein (24). In the present study, we used the cytoplasmically located N-terminus of Pgp as bait with an expectation of identifying novel proteins that regulate Pgp trafficking as this region is essential for the insertion of Pgp into the ER. As shown in this report, the yeast two-hybrid analysis identified Bap29varP as the interacting protein with the N-terminus of Pgp. Since Bap29 and its related Bap31 proteins are now known to regulate the trafficking of several membrane proteins including CFTR, which were mentioned in the introduction, we focused on Bap29varP with the expectation that this ER protein regulates Pgp trafficking. Clearly, Bap29varP is another isoform of Bap29 as its cDNA fragment obtained in the yeast two-hybrid analysis contained a cDNA encoding 34 residue peptide, stop codon and poly A tail. Based on its homology with other forms of Bap29, we deduced the full-length of Bap29varP, which was supported by its PCR amplification of an identical DNA fragment and sequencing in many cancer cells (Fig. 3C). Bap29varP is a 303 amino acid long polypeptide and predictive algorithms suggest that it contains three TM segments located at residues 7-29, 44-63 and 103-123 and a cytoplasmically located 180 amino acid long C-terminus, similar to the topology of Bap29 and Bap31. The cytoplasmic location of the $\mathrm{C}$-terminus of Bap29varP provides an easy explanation of its interaction with the similarly localized $\mathrm{N}$-terminus of Pgp. To demonstrate the protein-protein interactions between Bap29varP and Pgp, we performed coimmunoprecipitation experiments using different detergents including dodecyl maltoside, octylglucoside to solubilize Pgp and Bap29varP. In these experiments, Pgp was poorly immunoprecipitated without the association with any detectable Bap29varP (datanot shown). It is therefore possible that the use of detergents in the solubilization of these integral membrane proteins results in the disruption of protein-protein interactions. To circumvent the problems in demonstrating the protein-protein interactions of two membrane proteins by IP, we carried out the yeast and mammalian two hybrid procedures.

The observations that the C-terminus of Bap29varP interacts specifically with the N-terminus of Pgp but not with either the C-terminus of Pgp or with an unrelated $\alpha$-ENaC subunit in the yeast two-hybrid system, pointed out that the binding of Bap29varP to Pgp represents a true interaction. As mentioned in Results, neither Bap31 nor Bap29 isoform 3 interacted with Pgp. In addition, the interaction tests carried out in HEK293 cells clearly pointed out that Bap29varP interacts with the N-terminus of Pgp (Fig. 3B). Furthermore, immunocytochemistry experiments clearly indicated that Bap29varP and Pgp are co-localized in the ER as well as in the intracellular vesicles (Fig. 5A). These observations together indicated that Bap29varP is a specific interacting protein of Pgp.

The data presented here indicate that Bap29varP plays an essential role in the post-translational modification of $\mathrm{Pgp}$, which is necessary for the forward trafficking of Pgp to the cell surface. We screened several cell lines to identify Bap29varP $\mathrm{vall}^{+}$cells, which resulted in the identification of HT-29 cells that are Bap29varP $\mathrm{v}^{+}$and $\mathrm{Pgp}^{-}$. On the other hand, MCF-7 cells did not contain Bap29varP. We utilized these two cell lines to understand the functional role of 
Bap29varP on Pgp. As shown in Fig. 6, Pgp does not exit ER in MCF-7 cells, which do not contain Bap29varP protein. On the other hand, Pgp reaches the cell surface of HT-29 cells, which express Bap29varP. As both MCF-7 and MCF$7 / \mathrm{Adr}^{\mathrm{R}}$ cells are derived from human breast cancers, it is reasonable to suggest that Pgp expressed in these cell lines would undergo similar post-translational modifications and migrate similarly in SDS-PAGE. Interestingly, Pgp expressed in the absence of Bap29varP is $\sim 160 \mathrm{kDa}$ protein, which is slightly smaller than the molecular mass of Pgp expressed in cells expressing Bap29varP. This suggested that most of the post-translational $\mathrm{N}$-glycosylation modification on Pgp occurs in the absence of Bap29varP. It is therefore possible that the smaller size of Pgp is due to incomplete $\mathrm{N}$-glycosylation processing in this cell line. This interpretation suggests Bap29varP is essential for the final stages of $\mathrm{N}$ glycosylation of Pgp. Since the $\sim 160 \mathrm{kDa}$ Pgp was not detectable at the cell surface, it is likely that complete posttranslational N-glycosylational modification is a prerequisite for the cell surface expression of Pgp. Although other posttranslational modifications such as phosphorylation are likely, the observations of Wagenaar and co-workers (29) and more recently Ambudkar and his co-workers (30), who have shown that $\mathrm{N}$-glycosylation although not necessary for the function of Pgp, is however essential for the proper routing and stability of Pgp at the cell surface. Taken together, these observations suggest that Bap29varP is essential for the proper post-translational $\mathrm{N}$-glycosylation modification of Pgp in the ER and subsequent trafficking to the PM.

In summary, we identified a new isoform of Bap29 as an interacting protein with Pgp, which is involved in the regulation of Pgp trafficking to the PM. These observations bear an impact in an important way on the modalities in reversing the MDR in cancers. For instance, it is conceivable that disruption of Bap29varP expression and/or function in $\mathrm{Pgp}^{+}$cancers could potentially prevent the cell surface expression of Pgp, leading to the resensitization of MDR cancers to chemotherapy.

\section{Acknowledgments}

This work was supported by grants from the National Institutes of Health (CA106625). The laboratory is also supported by funds from the Department of Defense (BC046411) and the Women's Health Research Institute, TTUHSC. We thank Drs. Prem Kumar Gupta and Rashmi Krishnapuram for useful comments on the manuscript.

\section{References}

1. Annereau JP, Szakacs G, Tucker CJ, Arciello A, Cardarelli C, Collins J, Grissom S, Zeeberg BR, Reinhold W, Weinstein JN, Pommier Y, Paules RS and Gottesman MM: Analysis of ATPbinding cassette transporter expression in drug-selected cell lines by a microarray dedicated to multidrug resistance. Mol Pharmacol 66: 1397-1405, 2004.

2. Ueda K, Cardarelli C, Gottesman MM and Pastan I: Expression of a full-length cDNA for the human 'MDR1' gene confers resistance to colchicine, doxorubicin and vinblastine. Proc Natl Acad Sci USA 84: 3004-3008, 1987.

3. Choi KH, Chen CJ, Kriegler M and Roninson IB: An altered pattern of cross-resistance in multidrug-resistant human cells results from spontaneous mutations in the mdr1 (P-glycoprotein) gene. Cell 53: 519-529, 1988.
4. Chen CJ, Chin JE, Ueda K, Clark DP, Pastan I, Gottesman MM and Roninson IB: Internal duplication and homology with bacterial transport proteins in the mdr1 (P-glycoprotein) gene from multidrug-resistant human cells. Cell 47: 381-389, 1986.

5. Hrycyna CA, Airan LE, Germann UA, Ambudkar SV, Pastan I and Gottesman MM: Structural flexibility of the linker region of human P-glycoprotein permits ATP hydrolysis and drug transport. Biochemistry 37: 13660-13673, 1998.

6. Germann UA, Willingham MC, Pastan I and Gottesman MM: Expression of the human multidrug transporter in insect cells by a recombinant baculovirus. Biochemistry 29: 2295-2303, 1990.

7. Sauna ZE and Ambudkar SV: About a switch: how P-glycoprotein $(\mathrm{ABCB} 1)$ harnesses the energy of ATP binding and hydrolysis to do mechanical work. Mol Cancer Ther 6: 13-23, 2007.

8. Sharom FJ: Shedding light on drug transport: structure and function of the P-glycoprotein multidrug transporter (ABCB1). Biochem Cell Biol 84: 979-992, 2006.

9. Schamel WW, Kuppig S, Becker B, Gimborn K, Hauri HP and Reth M: A high-molecular-weight complex of membrane proteins BAP29/BAP31 is involved in the retention of membranebound IgD in the endoplasmic reticulum. Proc Natl Acad Sci USA 100: 9861-9866, 2003.

10. Breckenridge DG, Nguyen M, Kuppig S, Reth M and Shore GC: The procaspase- 8 isoform, procaspase- $8 \mathrm{~L}$, recruited to the BAP31 complex at the endoplasmic reticulum. Proc Natl Acad Sci USA 99: 4331-4336, 2002.

11. Paquet ME, Cohen-Doyle M, Shore GC and Williams DB: Bap29/31 influences the intracellular traffic of MHC class I molecules. J Immunol 172: 7548-7555, 2004.

12. Adachi T, Schamel WW, Kim KM, Watanabe T, Becker B, Nielsen PJ and Reth M: The specificity of association of the IgD molecule with the accessory proteins BAP31/BAP29 lies in the IgD transmembrane sequence. EMBO J 15: 1534-1541, 1996.

13. Annaert WG, Becker B, Kistner U, Reth M and Jahn R: Export of cellubrevin from the endoplasmic reticulum is controlled by BAP31. J Cell Biol 139: 1397-1410, 1997

14. Ng FW, Nguyen M, Kwan T, Branton PE, Nicholson DW, Cromlish JA and Shore GC: p28 Bap31, a Bcl-2/Bcl-XL- and procaspase-8-associated protein in the endoplasmic reticulum. J Cell Biol 139: 327-338, 1997.

15. Ng FW and Shore GC: Bcl-XL cooperatively associates with the Bap31 complex in the endoplasmic reticulum, dependent on procaspase-8 and Ced-4 adaptor. J Biol Chem 273: 3140-3143, 1998.

16. Kim KM, Adachi T, Nielsen PJ, Terashima M, Lamers MC, Kohler $\mathrm{G}$ and Reth M: Two new proteins preferentially associated with membrane immunoglobulin D. EMBO J 13: 3793-3800, 1994.

17. Spiliotis ET, Manley H, Osorio M, Zuniga MC and Edidin M: Selective export of MHC class I molecules from the ER after their dissociation from TAP. Immunity 13: 841-851, 2000.

18. Stojanovic M, Germain M, Nguyen M and Shore GC: BAP31 and its caspase cleavage product regulate cell surface expression of tetraspanins and integrin-mediated cell survival. J Biol Chem 280: 30018-30024, 2005

19. Lambert G, Becker B, Schreiber R, Boucherot A, Reth M and Kunzelmann K: Control of cystic fibrosis transmembrane conductance regulator expression by BAP31. J Biol Chem 276: 20340-20345, 2001

20. Szczesna-Skorupa E and Kemper B: BAP31 is involved in the retention of cytochrome P450 2C2 in the endoplasmic reticulum. J Biol Chem 281: 4142-4148, 2006.

21. Rao US and Nuti SL: Identification of two different states of P-glycoprotein in its catalytic cycle: role of the linker region in the transition between these two states. J Biol Chem 278: 46576-46582, 2003.

22. Nuti SL, Mehdi A and Rao US: Activation of the human Pglycoprotein ATPase by trypsin. Biochemistry 39: 3424-3432, 2000.

23. Nuti SL and Rao US: Proteolytic cleavage of the linker region of the human P-glycoprotein modulates its ATPase function. J Biol Chem 277: 29417-29423, 2002.

24. Rao PS, Mallya KB, Srivenugopal KS, Balaji KC and Rao US: RNF2 interacts with the linker region of the human P-glycoprotein. Int J Oncol 29: 1413-1419, 2006

25. Fairchild CR, Moscow JA, O'Brien EE and Cowan KH: Multidrug resistance in cells transfected with human genes encoding a variant P-glycoprotein and glutathione S-transferasepi. Mol Pharmacol 37: 801-809, 1990. 
26. Rao PS, Govindarajan R, Mallya KB, West W and Rao US: Characterization of a new antibody raised against the NH2 terminus of P-glycoprotein. Clin Cancer Res 11: 5833-5839, 2005.

27. Mechetner EB and Roninson IB: Efficient inhibition of P-glycoprotein-mediated multidrug resistance with a monoclonal antibody. Proc Natl Acad Sci USA 89: 5824-5828, 1992.

28. Loo TW and Clarke DM: Correction of defective protein kinesis of human P-glycoprotein mutants by substrates and modulators. J Biol Chem 272: 709-712, 1997.

29. Schinkel AH, Kemp S, Dolle M, Rudenko G and Wagenaar E: $\mathrm{N}$-glycosylation and deletion mutants of the human MDR1 P-glycoprotein. J Biol Chem 268: 7474-7481, 1993.

30. Gribar JJ, Ramachandra M, Hrycyna CA, Dey S and Ambudkar SV: Functional characterization of glycosylationdeficient human P-glycoprotein using a vaccinia virus expression system. J Membr Biol 173: 203-214, 2000

31. Labialle S, Gayet L, Marthinet E, Rigal D and Baggetto LG: Transcriptional regulators of the human multidrug resistance 1 gene: recent views. Biochem Pharmacol 64: 943-948, 2002.

32. Skach WR, Calayag MC and Lingappa VR: Evidence for an alternate model of human P-glycoprotein structure and biogenesis. J Biol Chem 268: 6903-6908, 1993.
33. Skach WR and Lingappa VR: Amino-terminal assembly of human P-glycoprotein at the endoplasmic reticulum is directed by cooperative actions of two internal sequences. J Biol Chem 268: 23552-23561, 1993.

34. Skach WR and Lingappa VR: Transmembrane orientation and topogenesis of the third and fourth membrane-spanning regions of human P-glycoprotein (MDR1). Cancer Res 54: 3202-3209, 1994.

35. Al-Shawi MK, Polar MK, Omote H and Figler RA: Transition state analysis of the coupling of drug transport to ATP hydrolysis by P-glycoprotein. J Biol Chem 278: 52629-52640, 2003.

36. Sauna ZE, Smith MM, Muller $M$ and Ambudkar SV: Functionally similar vanadate-induced 8-azidoadenosine 5'[alpha-(32)P]Diphosphate-trapped transition state intermediates of human P-glycoprotin are generated in the absence and presence of ATP hydrolysis. J Biol Chem 276: 21199-21208, 2001.

37. Chen EY, Bartlett MC, Loo TW and Clarke DM: The DeltaF508 mutation disrupts packing of the transmembrane segments of the cystic fibrosis transmembrane conductance regulator. J Biol Chem 279: 39620-39627, 2004.

38. Kartner N, Augustinas O, Jensen TJ, Naismith AL and Riordan JR Mislocalization of delta F508 CFTR in cystic fibrosis sweat gland. Nat Genet 1: 321-327, 1992. 